\title{
Picturing the Membrane-assisted Choreography of Cytochrome P450 with Lipid Nanodiscs
}

Carlo Barnaba ${ }^{[a]}$ and Ayyalusamy Ramamoorthy ${ }^{*[a]}$

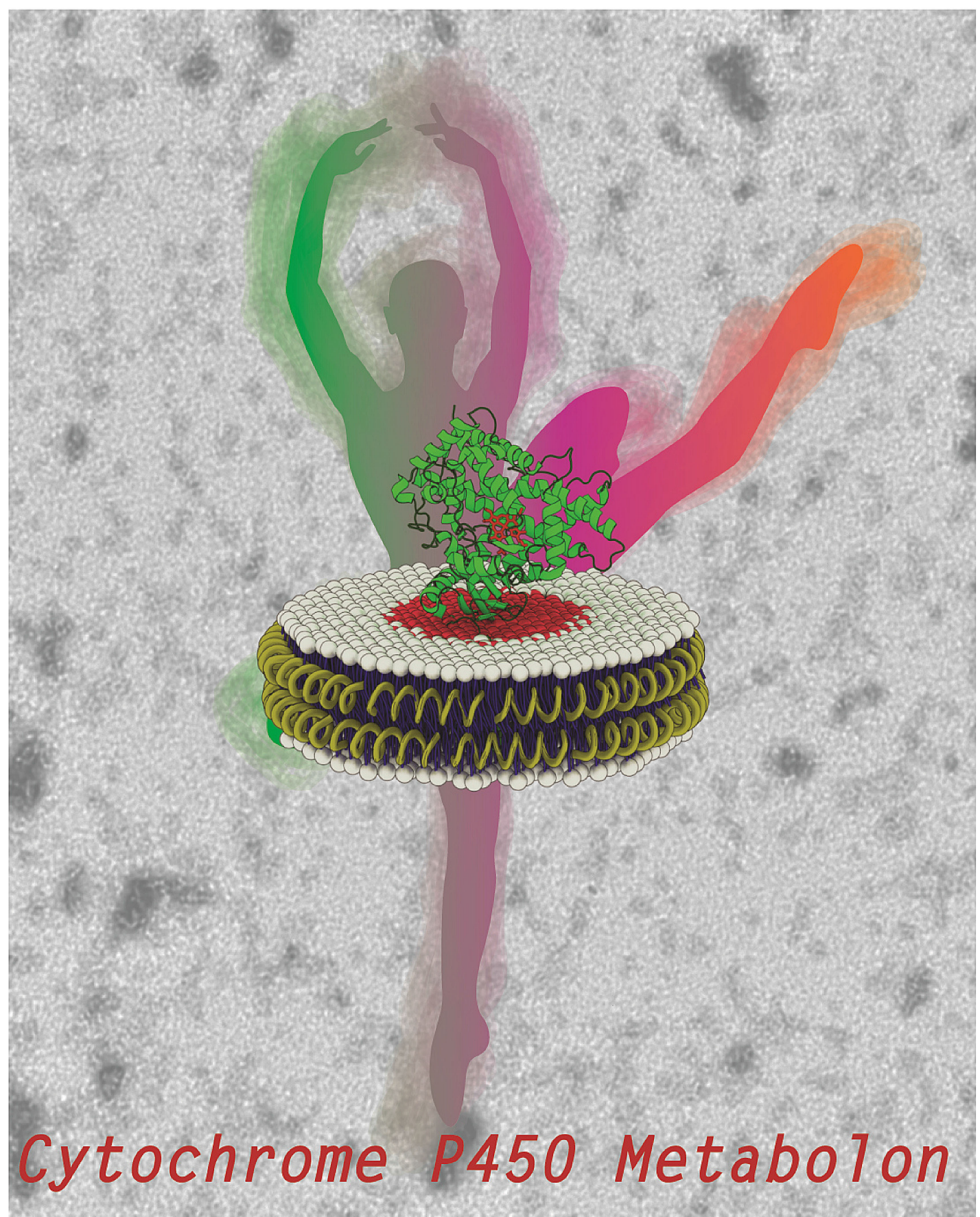


Abstract: Cytochrome P450, a family of monooxygenase enzymes, is organized as a catalytic metabolon, and requires enzymatic partners as well as environmental factors that tune its complex dynamic activity. P450 and its reducing counterparts are membrane-bound proteins which are believed to dynamically interact to form functional complexes. Increasing experimental evidence signifies the role (s) of protein-lipid interactions in P450's catalytic function and efficiency. The challenges posed by the membrane have severely limited high-resolution understanding of the molecular interfaces of these interactions. Nevertheless, recent NMR studies have provided piercing insights into the dynamic structural interactions that enable the function of P450. In this review, we will discuss different biomimetic approaches relevant to unveil molecular interplays at the membrane, focusing on our recent work on lipid-nanodiscs. We also highlight the need to expand the use of nanodiscs, and the power of a combination of cutting-edge solution and solid-state NMR techniques, to study the dynamic structures of P450 as well as other membrane-proteins.

\section{Introduction}

Cytochrome P450 (P450) enzymes are a ubiquitous family of monooxigenases responsible for the catalysis of numerous reactions of both physiological and biotechnological importance. ${ }^{[1]}$ They are widely diffused in the prokaryotic and eukaryotic worlds, including plants, fungi and animals. In plants, P450s are the key components in numerous processes of general and specialized metabolism, including the biosynthesis of plant hormones, and in cell wall biosynthesis as well as in the biosynthesis of specialized compounds involved in plant defense. ${ }^{[2]}$ In mammalians, more than 40 gene families have been recognized; similar to plants, mammalian P450s participate in the biosynthesis of steroid hormones, and are pivotal in cholesterol homeostasis and other signaling processes. ${ }^{[1]}$ In humans, 58 distinct P450s genes have been identified, and 13 isoforms are involved in the metabolism of drugs and xenobiotics, $^{[3]}$ consolidating P450's research as major interest for pharmacology, pharmacogenetics and drug discovery areas. Eukaryotic P450s are membrane proteins, bound to the membrane of the endoplasmic reticulum (ER) or mitochondria, ${ }^{[4-5]}$ whereas most bacterial P450s are soluble. ${ }^{[1]}$ In humans, the catalytic action of cytochrome P450s occurs via interprotein electron transfer (ET) between the oxygenase (P450) and the reducing counterparts cytochrome P450-reductase (CPR) or adrenodoxin reductase for ER or mitochondrial systems, respectively. Both electron transferases are membraneassociated proteins and use NADPH cofactor as reducing agent. $\mathrm{P} 450$ 's catalysis, including ligand binding and the electrontransfer steps, is orchestrated at the membrane interface, giving the lipid bilayer a vast importance that spans reaction efficiency, protein stability as well as protein subcellular organization. ${ }^{[1,6-7]}$ At the same time, the lipid membrane poses numerous challenges for both structural and kinetic studies. ${ }^{[6]}$ Unlike the intrinsic membrane proteins, the large-soluble domain containing P450 defies structural and physiologically-relevant functional investigations. In this review, we aim to provide back-

[a] Dr. C. Barnaba, Prof. A. Ramamoorthy

Biophysics and Department of Chemistry

University of Michigan

Ann Arbor, MI 48109-1055 (USA)

E-mail: ramamoor@umich.edu ground and an overview of P450's dynamics within the lipid membrane and their consequences on its structure and activity. We will discuss different biomimetic approaches relevant to unveil molecular interplays at the membrane, focusing on our recent work on peptide-based nanodiscs. ${ }^{[8]}$ Lastly, a perspective of the horizons of microsomal P450's research will be discussed.

\subsection{Structure and Function of Cytochrome P450}

Cytochrome P450s are $\sim 56 \mathrm{kDa}$ proteins consisting of $12 \alpha$ helices and anti-parallel $\beta$-sheets. ${ }^{[1]}$ The $\mathrm{N}$-terminus contains the transmembrane domain (TMD), which includes a signal-anchor sequence, a stretch of hydrophobic amino acids, followed by a basic sequence and a proline-rich sequence. ${ }^{[9-10]}$ The lower cleft of the cytosolic domain is also believed to interact with the membrane, through the so-called $\beta$-finger region and the $F-G$ loop (Figure 1a). This extended protein/membrane interface explains P450's modest lateral membrane mobility, as observed in both model ${ }^{[11-12]}$ and cell membranes. ${ }^{[10]}$ All $\mathrm{P} 450$ s possess an essential heme prosthetic group, stacked between the I and L helices. The heme is bound to the protein skeleton via a coordination of Fe-thiolate bound with the proximal cysteine residue. The heme sits in a relatively large pocket, surrounded by hydrophobic amino acid residues to accommodate hydrophobic substrates. The P450's metabolic cycle proposed in 1966 by Omura and coworkers, ${ }^{[13]}$ except for a few adjustments, is still valid (Figure $1 \mathrm{~b}$ ). In the rest state, the oxidized $\mathrm{Fe}^{3+}-\mathrm{P} 450$ is mainly in low-spin electronic configuration; substrate binding alters the spin state to high-spin, making the iron prone to be reduced $\left(\mathrm{Fe}^{2+}-\mathrm{P} 450\right)$.

$\mathrm{Fe}^{2+}-\mathrm{P} 450$ can then bind molecular oxygen and receive an additional electron to form the unstable hydroperoxyl intermediate known as Compound-O ( $C p d \quad 0$ ). The $\mathrm{O}_{2}^{-2}$ :porphyrin complex reacts with the surrounding protons to form the catalytically active oxyferryl intermediate, known as Compound-I ( $C p d$ I). The Fe-ligated oxygen atom is then transferred to the substrate, and the hydroxylated product is then released. Beside hydroxylation, P450s also catalyze a variety of other significant reactions, including $\mathrm{O}$ - and $\mathrm{N}$-demethylation, and epoxidation, although additional minor and unusual chemistries have been reported. ${ }^{[15]}$ 

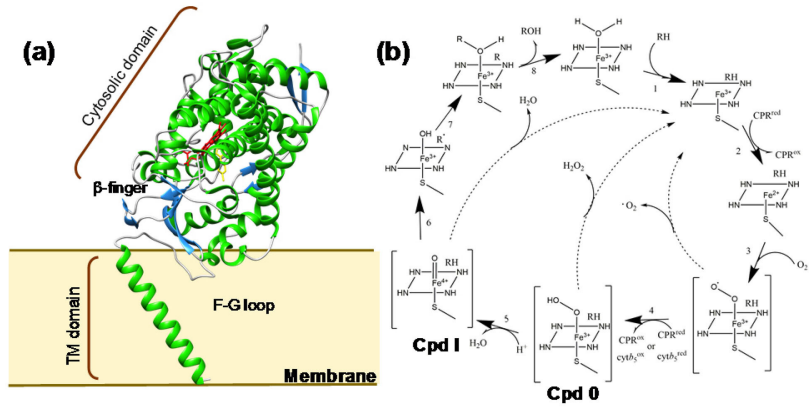

Figure 1. Structure and function of cytochrome P450. a) Membrane topology of cytochrome P450 (CYP2B4, PDB code is 1SUO). The transmembrane (TM) domain was built in Chimera using the sequence retrieved in Uniprot database. Based on oriented solid-state NMR experiments, the transmembrane domain spans the lipid bilayer and is tilted $\sim 15^{\circ}$ away from the lipid bilayer normal (the angle is accentuated in the picture). ${ }^{[14]}$ Residues of the cytosolic domain also interact with the lipid bilayer. b) The catalytic cycle of cytochrome P450. The binding of substrate R-H (1) causes a decrease in the redox potential of about $100 \mathrm{mV}$, which allows the first electron transfer from cytochrome P450-reductase (CPR) (2). The reduction of $\mathrm{Fe}^{3+}$ to $\mathrm{Fe}^{2+}$ in P450 enables $\mathrm{O}_{2}$ binding (3) and acceptance of a second electron from either CPR or cytochrome $b_{5}$ (cyt $b_{5}$ ) (4) to form a hydroperoxyl intermediate known as Compound- 0 . The $\mathrm{O}_{2}^{-2}$ complex reacts with surrounding protons to form the highly reactive oxyferryl intermediate, also known as Compound-I (5). The Fe-ligated $\mathrm{O}$ atom (6) is transferred to the substrate forming a hydroxylated form of the substrate $(\mathrm{ROH})(7)$. The product $(\mathrm{ROH})$ is finally released (8) by replacing it by a water molecule. Three uncoupling reactions are shown as dashed lines, with the respective products: the autoxidation shunt $\left(\mathrm{O}_{2}^{-2}\right)$, the peroxide shunt $\left(\mathrm{H}_{2} \mathrm{O}_{2}\right)$, and the oxidase shunt $\left(\mathrm{H}_{2} \mathrm{O}\right)$ (adapted from Barnaba et al. $\left.{ }^{[6]}\right)$.

Several aspects of P450's catalytic cycle are entwined to the lipid membrane. ${ }^{[6]}$ P450s mostly catalyze reaction on lipophilic compounds, thus ligands' partition is likely shifted to the hydrophobic core of the lipid membrane, rather than to the cytosol. As a consequence, the migration of ligands towards the active site occurs via protein "hydrophobic channels" exposed to the membrane leaf, as pointed out by several authors. ${ }^{[16-17]} \mathrm{A}$ few isoforms, including the major drug-metabolizing CYP3 A4, possess an allosteric site facing the membrane, ${ }^{[18]}$ a finding that has a particular significance for drugdrug interactions. ${ }^{[19-21]}$ The catalytic function of cytochrome P450s require two electrons: both electrons can directly be transferred by CPR; or after the direct transfer of the first electron by CPR, the second electron can be first transferred from CPR to cytochrome $b_{5}$ (cyt $b_{5}$ ) and then from cyt $b_{5}$ to P450.
The thermodynamic force driving the electron transfer is the difference in redox potential between the donor (CPR or $\mathrm{cyt}_{5}$ ) and the acceptor (P450) in their metabolically active forms. For the redox couple $C P R^{2-}: P 450-\mathrm{Fe}^{2+}$, the redox potential difference is about $-300 \mathrm{mV}$ in the absence of a substrate. ${ }^{[22]} \mathrm{Cyt} b_{5}$ has a higher redox potential $(+25 \mathrm{mV})$, which makes it unable to transfer the first electron to P450. ${ }^{[23]}$ The ternary enzymatic system is collectively known as "P450 metabolon", since the high interdependence between the different enzymes in performing the catalysis. ${ }^{[7]} \mathrm{CPR}$ and $c y t b_{5}$ are also membraneanchored proteins, although their topology differs from that of $\mathrm{P} 450$. CPR is a large $(\sim 78 \mathrm{kDa})$ flavoprotein with a flavin adenine dinucleotide (FAD) and a flavin mononucleotide (FMN) domains connected via a hinge region. CPR contains a highly disordered region in the $\mathrm{N}$-terminus preceding the helical transmembrane domain; CPR's high lateral mobility is probably related to its different topology compared to $\mathrm{P} 450 .{ }^{[11-12]} \mathrm{Cyt}_{5}$ is instead a small $(\sim 15 \mathrm{kDa})$ hemeprotein, whose full-length membranebound high-resolution structure and topology were determined by our group using a combination of solution and solid-state NMR techniques. ${ }^{[24]}$ We revealed that, similar to $\mathrm{P} 450$, cyt $b_{5}$ is indeed a bitopic membrane protein with a cytosolic domain not interacting with the membrane surface, and exhibit fast time scale of motion ( $\sim$ microsecond or faster). ${ }^{[25-26]}$ Our solidstate NMR experiments revealed that the transmembrane domains of both cyt $b_{5}$ and P450 were found to be helical with $\sim 15^{\circ}$ tilt relative to the lipid bilayer normal, and undergo a very slow time scale of motion (<milliseconds). ${ }^{[14,25]}$ In addition, our recent dynamic nuclear polarization (DNP) based magic angle spinning (MAS) solid-state NMR experiments revealed that the two transmembrane domains interact in the $\mathrm{cyt}_{5}-\mathrm{P} 450 \mathrm{com}$ plex and have a cross at the leucine zipper region of cyt $b_{5 \cdot}{ }^{[14]}$ Our solution NMR experiments have revealed the interacting interface in the soluble domains of the cyt $b_{5}-\mathrm{P} 450$ complex. ${ }^{[24]}$ The interactions between P450 and the redox counterparts are dynamic in nature, ${ }^{[12]}$ and allow the respective prosthetic moieties to be in a spatial vicinity suitable for ET to occur.

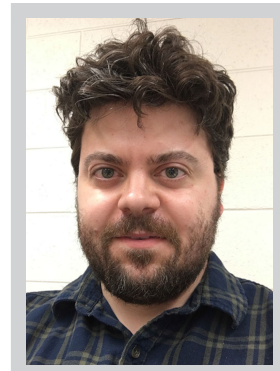

Dr. Carlo Barnaba received his B.Sc. and M.Sc. (cum laude) at the Universita' degli Studi di Parma (Italy). In 2016, he earned a PhD in Chemistry at Washington State University (WA) under the supervision of James A. Brozik and Jeffrey P. Jones, where he employed single-molecule spectroscopy to study protein-lipid and protein-protein interactions in the cytochrome P450's metabolon. He is a post-doctoral fellow in the Ramamoorthy's lab at the University of Michigan since 2017. His research interests involve the biochemistry and biophysics of redox metabolons, with a focus on membrane-related dynamics.

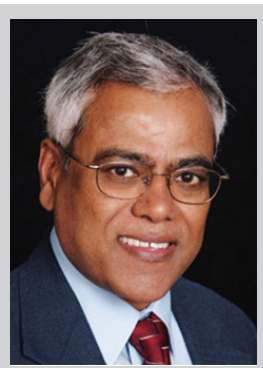

Prof. Ayyalusamy Ramamoorthy is Robert W. Parry Collegiate Professor of Chemistry and Biophysics at the University of Michigan, where he has been a faculty member since 1996. His current research interests include the development and applications of NMR spectroscopy to study the structure, dynamics, and function of membrane protein complexes, amyloid proteins, nanomaterials, and biomineralized tissues. Details on his current research can be found at http://www.umich.edu/ ramslab. 


\section{Nanodiscs as a Platform for P450's Biophysics}

\subsection{Early Membrane Mimetics for P450's Biochemical Studies}

Since the dawn of P450's research it was unequivocal that a lipid-like environment was vital for both stability and activity of cytochrome $\mathrm{P} 450$, in spite of studies that continue to avoid the use of membrane due to the associated complexities. Pioneering studies by Ingelman-Sundberg and Coon established that a lipid environment is essential for CPR-mediated ET and thus cytochrome P450 catalysis, and protein/lipid interactions are governed by the electrostatic nature of lipids. ${ }^{[27-29]}$ Hence, parallel to biochemical studies, a conspicuous effort has been directed to develop membrane systems that could closely mimic the native lipid bilayer. Detergent-based micellar systems and liposomes have been broadly used for activity reconstitution, although our results and other groups have shown that in detergent P450 exists in the inactive P420 state. ${ }^{[24]}$ On the other hand, bicelles exhibited native-like folding and function for P450, and enabled high-resolution NMR studies. ${ }^{[30]}$ Although bicelles rendered far more stability for active P450, it was limited to days. Since the detergent or short-chain lipid molecules surrounding like a belt of the planar lipid bilayer in bicelles diffuse into the lipid bilayer, our results showed that P450's stability directly depend on the amount of lipids to detergent ratio. On the other hand, homogeneous ${ }^{[28]}$ and heterogeneous ${ }^{[31]}$ liposomal preparation have shown good metabolic activity performances compared to native microsomal extracts. Liposomes have also been extensively used for spectroscopic studies on protein-protein interactions of the metabolon. ${ }^{[6,32]}$ It is worth mentioning that beside a few examples, ${ }^{[33]}$ the composition of liposomes did not take the native membrane heterogeneity into account. The ER membrane is highly heterogeneous: it is mainly composed by zwitterionic phospholipids (75-85\%), with a discrete percentage of negatively charged components (10-15\%) including phosphatidylserine (PS) and phosphatidylinositol (PI). Surprisingly, a consistent body of literature has been sustained by spectroscopic and kinetic studies performed on membrane mimetics that are far to be representative of the ER membrane. To cite a few examples, several studies have been realized on highly-negatively charged baculovirus expression systems (also known as baculosomes), ${ }^{[34]}$ whose cell membrane contains up to $23 \% \mathrm{PI}$ and $36 \% \mathrm{PE}^{[35]}$ Others ${ }^{[34,36]}$ have used liposomal preparations enriched with phosphatidic acid (PA), which is known to segregate in large microdomains. Although useful for metabolic and structural purposes, the authors consider that the extrapolation of more profound biological considerations such as subcellular protein organization and protein-protein dynamics - from reconstitution systems that are not representative of the ER membrane can be biased by artifact and should be avoided. Only very recently such ambiguity has been finally recognized, and protein-membrane and protein-protein dynamics in the P450's metabolon have been approached at both ensemble and single-molecule level using mimetics closely resembling the ER membrane. ${ }^{[11-12,37]}$ High-resolution in-cell solid-state NMR investigation was recently reported to probe the structural features and membrane interaction of cyt $b_{5}$, and demonstrated that the use of lipid vesicles provided near-native results for the cytochrome protein. ${ }^{[38]}$

Removal of the transmembrane domain of cytochrome P450 results in a solubilized form of the enzyme that - although only marginally active - has been found to be suitable for crystallization studies. For decades, it has been a useful strategy and allowed the crystallization of several P450s, including 22 human isoforms. ${ }^{[39]}$ On the other hand, full-length structural studies of P450 have been overwhelming for many decades; the structure of the yeast CYP51 in detergents by Monk et al. ${ }^{[40]}$ is the only full-length P450 X-ray structure to date, albeit the lack of a lipid bilayer in the sample. Structural NMR is the "trojan horse" that has allowed some of the most important breakthroughs in $\mathrm{P}^{4} 50$ 's research, ${ }^{[25,41-42]}$ uncovering critical information regarding both protein-protein and protein-membrane information that were previously secluded. Our laboratory has led the use of both solution and solid-state NMR techniques to study reconstituted P450, from the early works on magnetically-aligned bicelles ${ }^{[43-44]}$ to our more recent efforts using sophisticated nanodiscs. ${ }^{[8,45-46]}$ The information provided by studies on full-length protein in native-like environments have opened new opportunities and overcome challenges in biochemical and pharmacological facets of P450's research, and concurrently abet novel questions related to the organization of the metabolon in the cellular membrane.

\subsection{From MSP to Peptide-based Lipid Nanodiscs}

Nanodiscs are lipid bilayer patches surrounded by an amphiphilic belt which takes inspiration from plasma lipoproteins. ${ }^{[4]}$ Since their introduction, ${ }^{[48-49]}$ nanodiscs have imposed themselves as the "gold standard" for lipid membrane mimetic, for both kinetic and structural studies. ${ }^{[47]}$ Three generations of nanodiscs have succeeded over time, with the major differences being the surrounding belt. The membrane-scaffold protein (MSP) nanodiscs were first introduced, being directly inspired by plasma apolipoproteins (apoA-I), which is the major protein component of high-density lipoproteins. MSP and its derivatives are large proteins (200-400 aa) that spontaneously form nanodiscs with several phospholipids; dimyristoylphosphatidylcholine (DMPC) and 1-palmitoyl-2-oleoyl-sn-glycero-3-phosphocholine (POPC) nanodiscs are the most used for structural studies. However, the rigidity provided by such a long amino acid chain makes obligate the co-presence of detergent (i.e. sodium cholate) for facilitating protein reconstitution. Detergents are then removed by dialysis and homogeneous nanodiscs preparations are generally attained. ${ }^{[47-49]}$ Later on, it has been noticed that mimetic of fragment (and designed) amphipathic peptides of apoA-I can also spontaneously self-assemble with lipids to form nanodiscs, with the following advantages: i) the size of nanodiscs is adjustable by varying the lipid:peptide molar ratio, and ii) detergent is not required for protein reconstitution. Among such peptides, we have successfully demonstrated that 
$22 \mathrm{~A}$ and $4 \mathrm{~F}$ can be used for P450's metabolon reconstitution. ${ }^{[50]}$ $22 \mathrm{~A}$ is derived from the repeated $\alpha$-helix domain of apoA-I but it is not easy to form nanodiscs with varying lipid mixtures. $4 \mathrm{~F}$ was developed by Kariyazono et al. ${ }^{[51]}$ and has been shown to overcome some of the technical limitations of $22 \mathrm{~A}$, including high versatility in lipid composition and stability during the size-exclusion chromatography steps necessary for nanodiscs' purification. ${ }^{[45,52]}$ We will discuss more about the peptide-based nanodiscs in the next section. For the sake of completeness, it is worth mentioning the polymer-based nanodiscs, which are the third generation of these type of membrane mimetics. The styrene-maleic acid (SMA) copolymer was the first efficiently used to reconstitute membrane proteins. ${ }^{[53]}$ Recently, our group has synthesized other SMA-based polymers that are able to form lipid nanodiscs ( $<20 \mathrm{~nm}$ diameter in size) that can be used for solution NMR studies and macro-nanodiscs ( $>20 \mathrm{~nm}$ diameter up to $\sim 60 \mathrm{~nm}$ ) that can magnetically-align and enable solid-state NMR experiments. ${ }^{[54-56]}$ These novel SMA derivatives have been demonstrated to exhibit enhanced chemical and physical stability (against temperature, $\mathrm{pH}$, and divalent metal ions such as $\mathrm{Ca}^{2+}$ and $\mathrm{Mg}^{2+}$ ) compared to the original SMA. Our group has also recently demonstrated the nanodiscs formation by a new class of polymethacrylate copolymers, that are "styrene-free" and can be used for fluorescence and circular dichroism (CD) measurements. Therefore, these polymers can be used for the investigation of membrane-assisted amyloid aggregation and to trap transient toxic amyloid intermediates for biomedical applications. ${ }^{[57]}$

\section{P450-Lipid Interactions at Nanometric Scale}

Dissecting the roles of lipids on P450's function and stability has occupied several research groups across the last decades. An exhaustive scrutiny of those findings is beyond the scope of this work and has been the subject of recent reviews. ${ }^{[6,58]}$ Instead, we will trace a route that goes from the initial speculations on $\mathrm{P} 450$ 's organization in the membrane towards our recent findings ${ }^{[8]}$ on the extraordinary ability of P450 in modifying its surrounding lipid boundaries.

\subsection{Lipids and P450: Old Tale, New Twists}

Early quantitative measurements in rat microsomes revealed a surprisingly unbalanced stoichiometry between P450 and CPR, with P450 in 5-to-10 folds excess. ${ }^{[59]}$ Thus, to explain CPR's efficiency in electron delivery, several speculations were made, which can be summarized in two different theories. The first, proposed by Estabrook ${ }^{[60]}$ and adopted by others, ${ }^{[3,61]}$ lied in the supposed organization of P450's monomer in quaternary complexes or "clusters" served by the monomeric CPR. Another theory developed in the same period attributed the observed ET efficiency to the differences in protein lateral organization and mobility. ${ }^{[62]}$ This last model has been recently corroborated by single-molecule microscopy studies on CYP2C9 and CPR reconstituted in a ER biomimetic. ${ }^{[11-12]}$ Diffusion models are mainly sustained on protein mass transport and kinetics at the membrane surface, making it more attractive while more challenging in terms of experimental probing. More importantly, a "diffusion model" cannot omit the role played by the lipid constituents, making it more appealing for membrane biophysical investigation. As a matter of fact, after liposomes were established as mimetic to reconstitute microsomal P450CPR pair activity by Ingelman-Sundberg and Coon, ${ }^{[27-29]}$ Stier's group further explored the role of phospholipids in the monooxygenase functions, even suggesting a "specific requirement" of phosphatidylcholine (PC) and phosphatidylethanolamine (PE) for P450 and CPR activities, respectively. ${ }^{[63-64]}$ For $C P R$, the reason of such a molecular preference resides in the negative-curvature effect provided by $P E$ that may help to accommodate its relatively large cytosolic domain. Experiments performed with ${ }^{31} \mathrm{P}-\mathrm{NMR}$ on $\mathrm{P} 450$ and CPR purified from microsomal extracts showed residual PE and phosphatidylserine (PS) strongly bound to the protein. ${ }^{[65-66]}$ Since membrane protein purification requires high amounts of detergents, it is undoubtedly that those results can be biased by the type of detergent used and the experimental procedure. Notwithstanding, it is certain that a strong lipid-protein association can be established through weak bonds between specific polar residues and the lipid's polar head group. Given the mounting evidence that PS could benefit P450's catalysis, Guengerich's lab set the ternary mixture DLPC:DOPC:DLPS $(1: 1: 1 \mathrm{w} / \mathrm{w} / \mathrm{w})$ as the "standard" recipe for P450's activity reconstitution, at least for drug metabolism studies. ${ }^{[3]]}$ The appearance of MSP-nanodiscs did not significantly motivate research on exploring the effects of membrane composition, and most of the initial studies were focused on the protein stability and function in this novel mimetic. In fact, homogeneous DMPC and POPC nanodiscs served as predominant scaffold for countless studies on P450's kinetics and structure, and to date are still broadly used. It was until 2009 that Sligar's lab included a discrete amount of PS lipid in POPC MSP-nanodiscs to study the effect of a negatively-charged phospholipid on the P450-CPR couple, demonstrating a modulation of CPR redox potential that favoured electron transfer and thus catalysis. More recently, it has also been shown that the length of the lipid chain has a significant action on P450's thermal stability, but marginally on substrate binding kinetics. ${ }^{[67]}$ This is potentially due to a different orientation and immersion of the protein in the membrane, as already displayed by molecular dynamic simulations for several P450's isoforms, including the human drug metabolizers CYP3 A4 and CYP2C9. ${ }^{[58,68]}$

\subsection{Membrane, Spin-state, and Protein-Protein Interactions}

Nanodiscs composed of a heterogeneous membrane composition are achievable with both MSP and peptide-based nanodiscs. ${ }^{[37]}$ As mentioned above, peptide-based nanodiscs do not require detergent for protein reconstitution, avoiding tedious detergent removal steps and possible artefacts of reconstitution. ${ }^{[45,50,52]}$ A solution of isotropic nanodiscs has been well demonstrated to be suitable for high-resolution solution NMR 
based structural studies on embedded membrane proteins. ${ }^{[50]}$ We took advantage of these properties of peptide-based lipidnanodiscs by performing spectroscopic and structural studies on microsomal cytochrome P450 2B4 (CYP2B4) in DMPC, POPC and heterogeneous POPC:POPS nanodiscs at various phospholipid molar ratios. ${ }^{[52]}$ In all the considered lipid mixtures, we were able to form homogeneous nanodiscs preparations, that were able to monomerize CYP2B4 or $\mathrm{cyt} b_{5}$, as well as the protein-protein complex. Visible absorption spectroscopy on reconstituted nanodiscs demonstrated that membrane heterogeneity alters the spin-state equilibrium of P450's heme, an unexpected result that goes alongside with the already known effects of protein architecture, ligands and porphyrin electronics on spin multiplicity. ${ }^{[0]}$

In the rest state, heme spin's population is shifted towards low-spin (85-95\%); when in anionic POPS-enriched membrane, a significant shift to high-spin state was observed $(30 \%)$, which is linked to a higher redox potential. Addition of cyt $b_{5}$ is known to further push the equilibrium to the high-spin state that is favourable for catalysis. ${ }^{[71]}$ Surprisingly, we found that phospholipids boosted this conversion to the point that almost $100 \%$ conversion was obtained when $c y t b_{5}$ was added to P450 2B4 reconstituted in $4 \mathrm{~F}$-nanodiscs in the presence of a saturating substrate (Figure 2a). The spectroscopic studies were well
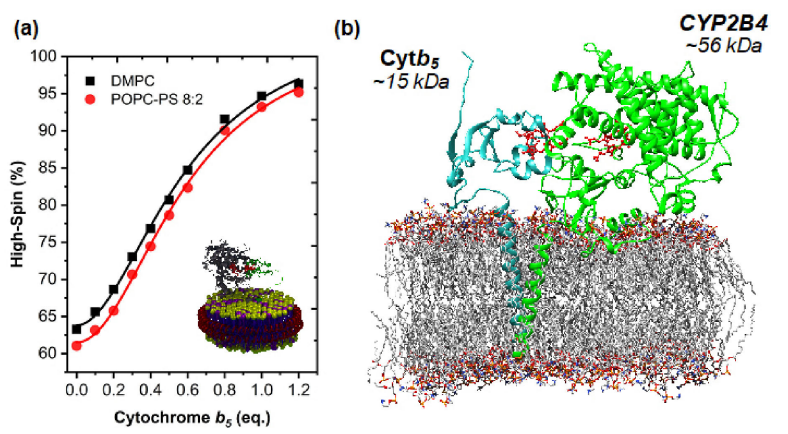

Figure 2. High-resolution investigation of P450-REDOX partner interaction. Functional reconstitution of the $\sim 70-\mathrm{kDa}$ CYP2B4-cyt $b_{5}$ complex in 4Fpeptide based lipid-nanodiscs. a) Titration of CYP2B4 with cyt $b_{5}$ in presence of excess ligand (benzphetamine) led to quasi-complete transition from lowto high-spin of the P450's heme in both DMPC and POPC:POPS (8:2) bilayer. b) Putative model of the CYP2B4:Cyt $b_{5}$ complex in lipid membrane, as obtained by HADDOCK simulation using experimental restraints from both X-ray crystallography and solution NMR (adapted from Ravula et al. ${ }^{[52]}$ and Yamamoto et al. ${ }^{[14]}$; lipid bilayer from Tieleman et al. ${ }^{[69]}$ ).

supported by solution NMR experiments on cyt $b_{5}$ titrated with CYP2B4. 2D ${ }^{15} \mathrm{~N}-{ }^{-1} \mathrm{H}$ HSQC-TROSY NMR spectra indicated a stronger and extensive interaction of $c y t b_{5}$ with CYP2B4, which was more pronounced when $20 \%$ of POPS was present. Several interacting residues were found, in both the lower and upper clefts of $c y t b_{5}$. Simulations performed using HADDOCK ${ }^{[72]}$ were used to build a putative structural model for the complex in nanodiscs, which reveals a close vicinity of the hemes for an efficient electron transfer (Figure 2b).

\subsection{Lipid-Exchange Experiment}

Inspired from the above-mentioned findings, but also from the earlier and genuine observations in microsomal preparations, ${ }^{[28-}$ 29,63,66] we decided to use nanodiscs as a "magnifying lens" for studying P450:lipid interactions. As mentioned above, in peptide-based nanodiscs in which the surrounding belt is made up of several peptides, and the nanodiscs undergo constant dynamic collisions between themselves. Specifically, lipids can "diffuse" from one nanodiscs to the others, a process known as "lipid exchange". ${ }^{[73-75]}$ While several factors can control the lipid exchange process, the lipid exchange has been found to be fast in peptide and polymer-based nanodiscs, whereas it is extremely slow in MSP nanodiscs. ${ }^{[76]}$ Mechanisms of lipid exchange between nanodiscs involve both monomer diffusion and fast collisional transfer. We were recently able to directly observe this phenomenon in 4F-DMPC nanodiscs by using high-speed AFM and ${ }^{31}$ P-NMR experiments. ${ }^{[75]}$ Lipid-exchange between nanodiscs and in general membrane biomimetics has emerged out to be very important for its implications in the equilibrium of small-molecules between lipoproteins (i.e. highdensity lipoproteins) and cell membranes, as well as for potential applications in membrane protein crystallography. ${ }^{[7,77]}$ This mass transport equilibrium can be potentially altered by small-molecules, peptide or protein that are able to specifically interact with membrane components, which is the basis of our experimental approach. 4F-peptides formed highly homogeneous nanodiscs when incubated with an ER lipid mixture that closely mimicked the native membrane composition. The ER membrane also contained the minor constituents sphingomyelin (SM, 7\%) and cholesterol (Ch, 4\%). We incubated CYP2B4 with excess nanodiscs and allowed the membrane insertion and lipid-exchange to occur overnight at a temperature above the $T_{m}$ of the lipid mixture. We used $\sim 8-\mathrm{nm}$ nanodiscs (Figure 3a), that roughly contain 35 to 40 lipids per leaf. ${ }^{[51]}$ The fraction of nanodiscs containing CYP2B4 was subsequently purified by size-exclusion chromatography (Figure $3 \mathrm{~b}$ ) and lipids quantified by ${ }^{31} \mathrm{P}-\mathrm{NMR}$ (phospholipids) or GC-MS (cholesterol).

\subsection{Formation of a Lipid Shell Surrounding P450}

As summarized in Figure $3 C$, a significant change in phospholipid composition in the fraction containing protein was observed. CYP2B4 was able to trap SM and $\mathrm{Ch}$, while partially excluding PE. A high local concentration of SM and $\mathrm{Ch}$ was associated with liquid-ordered $\left(I_{0}\right)$ regions of the lipid membrane, or the so-called raft domains ${ }^{[78]}$ Literature regarding the existence and the nature of lipid rafts has been controversial, but more recent evidence point out that domains in native cell membranes are smaller than previously found for model membrane $(<100 \mathrm{~nm})$, and are driven by both lipid-lipid and lipid-protein interactions. ${ }^{[79]}$ Rafts play important roles in cellular signalling and membrane trafficking, although visualization and characterization of rafts in cells is still challenging. ${ }^{[79]}$ Our membrane model was shown to be homogeneous over a 
(a)
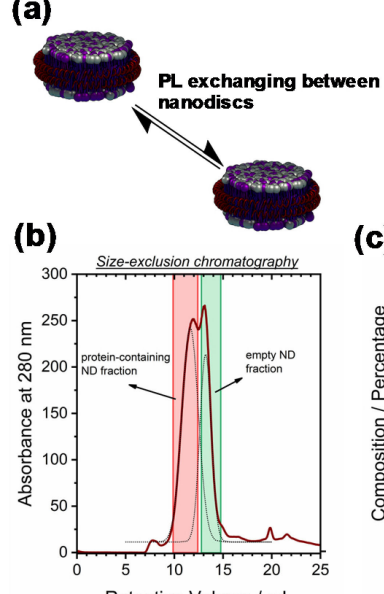

(d)

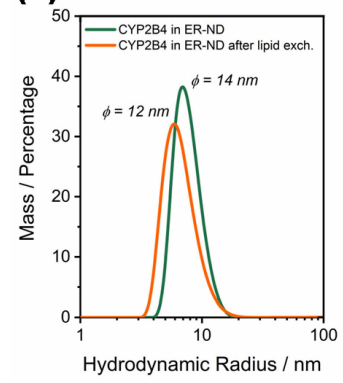

(c)
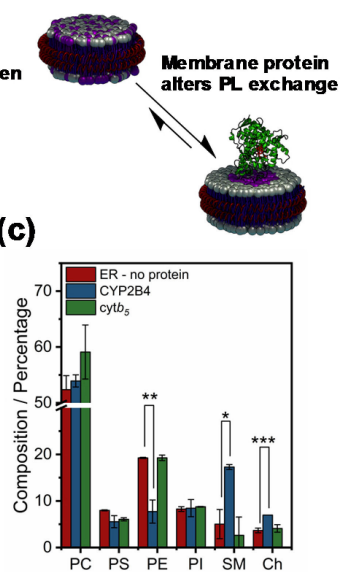

(e)

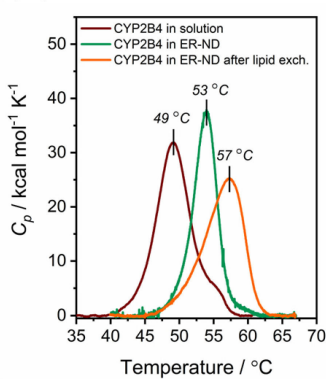

Figure 3. P450 preference for lipid-raft domains is revealed by peptide nanodiscs. Lipid-exchange experiment in 4F-ER nanodiscs allows the characterization of protein-induced "raft" formation. a) Schematic of the lipid-exchange experiment in peptide-based nanodiscs. In the absence of protein (left) the nanodiscs undergo phospholipid (PL) exchange via collisional transfer mechanism. The presence of a membrane protein (right) in a fraction of nanodiscs can perturb the mass equilibrium if the protein is able to specifically "retain" lipid components by weak protein-lipid interactions. b) Protein-containing nanodiscs can be purified by sizeexclusion chromatography. c) The amount of lipid and cholesterol is estimated by ${ }^{31} \mathrm{P}-\mathrm{NMR}$ and GC-MS chromatography, showing an enrichment of SM and Ch in CYP2B4-containing nanodisCs, with a depletion of PE. d) After lipid exchange, the diameter of a reconstituted nanodisc is reduced, indicating that the formation of a raft domain has consequences in the lateral distribution of lipid components. e) Differential scanning calorimetry of CYP2B4 in solution or in 4F-nanodiscs before and after lipid exchange show an increased thermal stability of the protein (adapted from Barnaba et al. $\left.{ }^{[8]}\right)$.

broad range of temperature, ${ }^{[11]}$ and fluorescence correlation spectroscopy (FCS) of 4F-ER nanodiscs showed analogue profile than $4 F-P O P C$ when an ordering-sensitive dye was used. ${ }^{[8]}$ This strongly evocates a role of the protein itself in segregating SM and $\mathrm{Ch}$. Few authors have hypothesized that membrane proteins can be encased in a "shell" of SM and Ch that can mediate the coupling between the transmembrane domain and the fluid lipid bilayer. ${ }^{[80-81]}$ The lipid shell is regulated by specific interactions between protein residues and phospholipid's polar head groups. ${ }^{[80]}$ Backes' work in rat microsomes found both P450 and CPR associated to detergent-resistant domains (DRM), which do not necessarily overlap with $I_{o}$ regions, given the questionable specificity of detergent in targeting specific phospholipids. ${ }^{[82]}$ On the other hand, $\mathrm{Ch}$ has been shown to inhibit CYP3 A4 activity, ${ }^{[83]}$ although it is likely that the rigidity conferred by the $I_{0}$ region can affect protein's

lateral diffusion and thus interprotein electron transfer. Another interesting finding is the partial depletion of $\mathrm{PE}$, which is known to negatively shape the membrane curvature, and it was shown to facilitate membrane binding and enhance the activity of human CYP3 A4, ${ }^{[84]}$ but not rabbit CYP1 A2 ${ }^{[85]}$ Likely, there is an isoform-specificity in the observed lipid segregation that deserves further investigation. The formation of a $l_{o}$ phase in a nanodisc is also reflected in a significant reduction in its diameter (Figure 3d). P450 is highly unstable outside the membrane and has a spontaneous and well-known tendency to aggregate to form non-specific oligomers. ${ }^{[28,64]}$ Furthermore, without any protectant agent like glycerol or detergents (i.e cholate or CHAPS), P450 easily converts to the inactive form (P420) in which the fifth coordination to the proximate cysteine is irreversibly lost. However, within the nanodisc, the protein is stable even at room temperature for a few days. ${ }^{[52]}$ Thermal stability of CYP2B4 is membrane-dependent, as demonstrated by us and others. ${ }^{[67]}$ The significant increase in both $T_{m}$ and $\triangle \mathrm{H}$ observed after lipid-exchange (Figure 3e) means that the tertiary structure of P450 is altered in the $I_{0}$ region.

We speculate that the association of P450 with the lipid shell can affect the conformation of the protein by the changes in the orientations of lipid-interacting residues. ${ }^{[80]}$ Similarly, fatty acid unsaturation and hence chains "packing" of lipids seemed to have analogue effect. ${ }^{[67]}$ When in the $I_{0}$ region, the phase transition is broader, probably indicating that the heme binding pocket is more open compared to the initial ER membrane, similar to what exerted by a substrate in CYP3 A4. ${ }^{[67,86]}$ For P450, a compound enters the active site and perturbs the heme's local electronic environment causing a shift of electronic configuration of $\mathrm{Fe}$ from low-spin to high-spin, with a consequent alteration of redox potential (from -300 to $-225 \mathrm{mV}$ ). ${ }^{[22]}$ We expected the lipid-assisted conformational change to have consequences in ligand binding, since in the general mechanism of binding an open form of the enzyme binds the substrate, and in doing so it folds around the substrate into a closed form. ${ }^{[87]}$ Indeed, the equilibrium constant for BHT (butylated hydroxytoluene) binding was altered by the nature of the surrounding lipids. The affinity was increased going from solution $(13 \mu \mathrm{M})$ to ER $(6 \mu \mathrm{M})$, while the lo region further increased the affinity equilibrium $(3 \mu \mathrm{M}){ }^{[8]}$ This is also accompanied with a dramatic increase in the low-to-high spin shift, which is a signature of differences in heme's exposure to solvent. Considering the importance of ligand binding on defining catalytic specificity, this membrane-dependent behaviour has direct consequence on the pharmacokinetics of lipophilic drugs, which represent a great majority of pharmacophores in the market. ${ }^{[8]}$

\subsection{P450's Specific Sphingomyelin Binding Region}

Cytochrome P450s is structurally fragile if not surrounded by a lipid-like environment. This peculiarity has dampened structural studies in membrane phase, but concurrently pushed researchers to use molecular dynamics simulations to shed light on protein-lipid interactions. ${ }^{[16,58,68]}$ We performed both all-atom 
(AA) and coarse-grained (CG) molecular dynamics simulations on CYP2B4 embedded in POPC or ER membranes. For the ER membrane, we use the original membrane composition, as well as the one enriched with SM and Ch. Briefly, both the transmembrane domain and the soluble domain presented different backbone dynamics in the lipid systems, with the protein being more restrained in the ER's $I_{o}$ domain (Figure 4a).

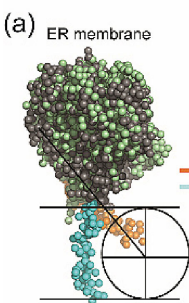

(c)

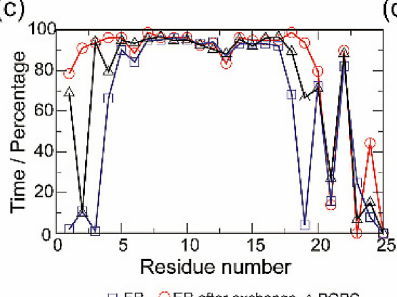

(b)

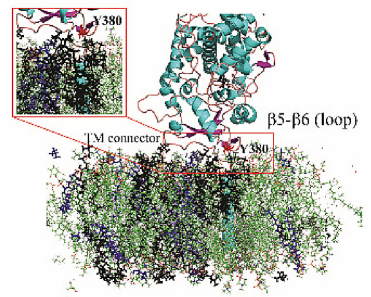

CYP2B4 in SM and Ch rich ER nanodiscs

(d)

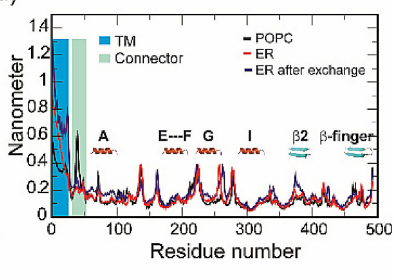

Figure 4. Membrane-bound structure of P450. Atomistic insights into the interaction between CYP2B4 and the ER membrane. a) Coarse-grained snapshot showing the TMD's orientation in POPC (left) and ER (right) nanodiscs at 0 (cyan) and $1 \mu$ s (orange). b) All-atom snapshot illustrating the interaction between CYP2B4 and ER membrane when enriched with SM and Ch. SM is shown in black. Specific residues are interacting with SM polar group (insert). c) Percentage of helicity per residue in the TMD during simulation time in different lipid systems. d) Root mean square fluctuations of CYP2B4 in different lipid systems (adapted from Barnaba et al. ${ }^{[8]}$ ).

Regions belonging to the proline-rich sequence and the $\beta$ finger motif presented higher rigidity compared to that in POPC, as from the RMS fluctuations, indicating a stronger interaction with membrane components. As a matter of fact, after $100 \mathrm{~ns}$ simulation, a few residues in the sequence spanning the connecting loop $\left({ }^{28} \mathrm{Gly}\right.$-Leu $\left.{ }^{40}\right)$ and the $\beta$-finger $\left.{ }^{375} \mathrm{Thr}-\mathrm{Il}^{382}\right)$ showed specific interactions with SM. Sequence alignment performed on several microsomal P450s showed that the membrane-interacting residues are conserved among P450's isoforms, which likely indicated that other $\mathrm{P} 450$ s possess similar ability to interact with lipids.

\section{Potential of Nanodiscs to Study Protein-Lipid Interactions}

\subsection{Current Strategies}

Specific interactions between membrane-associated proteins and membrane lipid components have captivated the attention of researchers for decades. ${ }^{[89]}$ Integral and membrane proteins account for roughly one-third of the full proteome ${ }^{[89]}$ and thus protein-lipid interactions are of paramount interest in pharmaceutical discovery. ${ }^{[90]}$ The complexity in terms of diversity and size of membrane components makes a detailed and unambiguous characterization of the interactions of transmembrane segments within a lipid bilayer highly challenging. ${ }^{[91-92]}$ Highresolution determination of molecular structure using diffraction methods requires high quality $3 \mathrm{D}$ crystals that are hard to obtain for membrane proteins, although significant breakthrough have been made by lipid-cubic phase crystallography and cryo-EM techniques. ${ }^{\text {[93-94] }}$

On the other hand, fluorescence-based techniques allow to visualize protein-lipid interaction in model membranes and cells. Model membrane systems - planar supported bilayers ${ }^{[95-}$ ${ }^{96]}$ and giant-unilamellar vesicles (GUVs), ${ }^{[97]}$ among others - have aided our understanding on the lipid organization of proteins in cell membranes. ${ }^{[98]}$ The advent of super-resolution imaging has impacted the microscopy field by overcoming the diffraction limit, ${ }^{[96]}$ and so solving structure that are separated by $\sim 200 \mathrm{~nm}$. However, a nanometric scale resolution is not accessible with microscopy techniques. Another common drawback of fluorescence-based techniques is the need of fluorescent lipid probes for visualization, that are not equivalent to their endogenous counterparts and can potentially create artefacts. ${ }^{[98]}$ Any attempt to understand physiological function of membrane-associated proteins must also be as precise and quantitative as possible. Along with the fact that extrapolating structural insights from fluorescence microscopy is not feasible, it is mandatory to develop new methodologies that can overcome these limitations and concurrently capture proteinmembrane interfaces at nanoscale resolution. ${ }^{[89]}$

\subsection{Opportunities from Peptide- and Polymer-Based Nanodiscs}

Detergent-free protein reconstitution and lipid-exchange dynamic are the major strengths of peptide or polymer-based nanodiscs. The existence of a mass equilibrium among individual nanodiscs make them prone for countless applications in structural biology and biophysics. We expect that our lipid-exchange strategy can potentially help answering some of the current questions in the biochemistry and biophysics of membrane-associated proteins and peptides. From the P450's side, although the transmembrane region is highly conserved, we cannot exclude an isoform-specific dependency on lipidprotein interactions. This is a fascinating hypothesis, that goes along with lipid signalling mechanisms that have been shown to provide the metabolic flexibility of the endoplasmic reticulum. ${ }^{[99]}$ As a matter of fact, given the heterogeneity of the P450's metabolon in terms of substrate catalysis, we anticipate that lipid-protein interactions could also play a role in the catalytic sorting. The role played by membrane and local lipid heterogeneity on P450's structure complements the recent finding on the conformational recognition triggered by substrate and inhibitors binding. ${ }^{[12,100]}$ The formation of electron transfer binary and ternary complexes with CPR and cyt $b_{5}$ could 
also be driven by specific interaction between the transmembrane domains of the proteins and the surrounding lipids. It is also known that the lipid membrane composition in the ER and other subcellular compartments is modulated by several physiological factors, including chronic disorders, inflammation and ageing. More than three decades ago it was already observed that an in vivo reduction of P450's activity parallel to a decline in the ratio of PC to PE and SM due to ageing. ${ }^{[101]} \mathrm{A}$ similar consideration is valid for mitochondria and associated monooxygenase system. ${ }^{[102]}$ Thus, applications for lipid-nanodiscs and lipid/protein interaction can transcend the mere interest in drug metabolism to a broader understanding of the function of the membrane oxygenase systems across different stages of life, in as much as during chronic pathologies. In fact, several other biological processes are governed by specific lipid-protein interactions, including amyloid aggregation, ${ }^{[103]}$ the insertion of pore-forming peptides, ${ }^{[104-105]}$ and virus entry, ${ }^{[106]}$ among others. The lipid-exchange experiment coupled with structural studies can potentially discern lipid-induced structural fluctuations that modulate such mechanisms.

\section{Final Remarks}

Earlier research in microsomal oxygenase systems had a genuine interest in protein-lipid interactions, which was unfortunately underestimated in the following decades. ${ }^{[6]}$ Some of the important discoveries of the last decade in P450 related research have foundations on those pioneering observations. Indeed, as from the Proceedings of the $3^{\text {rd }}$ International Symposium in Microsomes and Drug Oxidation in 1977, Stier and coworkers already noticed a rigid lipid area surrounding P450, with a partial depletion of PE and enrichment of SM. ${ }^{[107]}$ Forty-years later, those cautious speculations were ultimately and quantitatively demonstrated by our group using sophisticated tools. The last decade has seen a progressive interest on the role played by membrane in the catalysis of P450s, as many studies and reviews have been published. Nanodiscs have certainly boosted the experimental capabilities, although the technical improvements of structural techniques - in particular solution and solid-state NMR - and their applications to functional membrane systems cannot be ignored. Particularly, the use of peptide or polymer based nanodiscs (and the weakly-aligned macro-nanodiscs) for solution NMR and the unique magnetically-aligned macro-nanodiscs for solid-state NMR experiments bound to expand the power of NMR applications to study a large range of membrane proteins and membrane-bound protein-protein complexes. While the sophisticated DNP experiments can be used to overcome the challenges faced by the poor sensitivity, the rapidly advancing proton-detected and ultrafast-MAS techniques will also be valuable to obtain high-resolution insights into protein-protein and protein-substrate interactions. ${ }^{[108-121]}$ We also expect the macro-nanodiscs to be highly valuable for studies using cryoEM and diffraction experiments. We hope that more researchers will be attracted by the unanswered fundamental questions to fully understand the organization and function of membranebound P450s.

\section{Acknowledgements}

This study was supported by NIH (GM084018 to A. R.).

\section{Conflict of Interest}

The authors declare no conflict of interest.

Keywords: cytochrome P450 - nanodiscs - membrane rafts lipid exchange $\cdot$ endoplasmic reticulum

[1] P. R. O. De Montellano, Cytochrome P450: structure, mechanism, and biochemistry, Springer Science \& Business Media, 2005.

[2] C. Weitzel, H. T. Simonsen, Phytochem. Rev. 2013, 14, 7-24.

[3] U. M. Zanger, M. Schwab, Pharmacol. Ther. 2013, 138, 103-141.

[4] P. A. Williams, J. Cosme, V. Sridhar, E. F. Johnson, D. E. McRee, Mol. Cell 2000, 5, 121-131.

[5] T. Omura, Chem.-Biol. Interact. 2006, 163, 86-93.

[6] C. Barnaba, K. Gentry, N. Sumangala, A. Ramamoorthy, F1000Research 2017, 6, 662.

[7] J.-E. Bassard, B. L. Møller, T. Laursen, Curr. Mol. Biol. Rep. 2017, 3, 37-51.

[8] C. Barnaba, B. R. Sahoo, T. Ravula, I. G. Medina-Meza, S. C. Im, G. M. Anantharamaiah, L. Waskell, A. Ramamoorthy, Angew. Chem. Int. Ed. 2018, 57, 3391-3395; Angew. Chem. 2018, 130, 3449-3453.

[9] E. Szczesna-Skorupa, K. Ahn, C. D. Chen, B. Doray, B. Kemper, J. Biol. Chem. 1995, 270, 24327-24333.

[10] E. Szczesna-Skorupa, C. D. Chen, S. Rogers, B. Kemper, Proc. Natl. Acad. Sci. USA 1998, 95, 14793-14798.

[11] C. Barnaba, M. J. Martinez, E. Taylor, A. O. Barden, J. A. Brozik, J. Am. Chem. Soc. 2017, 139, 5420-5430.

[12] C. Barnaba, E. Taylor, J. A. Brozik, J. Am. Chem. Soc. 2017, 139, 1792317934.

[13] T. Omura, E. Sanders, R. Estabrook, D. Cooper, O. Rosenthal, Arch. Biochem. Biophys. 1966, 117, 660-673.

[14] K. Yamamoto, M. A. Caporini, S. C. Im, L. Waskell, A. Ramamoorthy, Sci. Rep. 2017, 7, 4116.

[15] E. M. Isin, F. P. Guengerich, Biochim. Biophys. Acta Gen. Subj. 2007 1770, 314-329.

[16] V. Cojocaru, P. J. Winn, R. C. Wade, Biochim. Biophys. Acta Gen. Subj. 2007, 1770, 390-401.

[17] K. Berka, T. Hendrychova, P. Anzenbacher, M. Otyepka, J. Phys. Chem. A 2011, 115, 11248-11255.

[18] C. Barnaba, S. C. Humphreys, A. O. Barden, J. P. Jones, J. A. Brozik, J. Phys. Chem. B 2016, 120, 3038-3047.

[19] I. G. Denisov, Y. V. Grinkova, J. L. Baylon, E. Tajkhorshid, S. G. Sligar, Biochemistry 2015, 54, 2227-2239.

[20] W. M. Atkins, Annu. Rev. Pharmacol. Toxicol. 2005, 45, 291-310.

[21] M. Shou, J. Grogan, J. A. Mancewicz, K. W. Krausz, F. J. Gonzalez, H. V. Gelboin, K. R. Korzekwa, Biochemistry 1994, 33, 6450-6455.

[22] S. G. Sligar, D. L. Cinti, G. G. Gibson, J. B. Schenkman, Biochem. Biophys. Res. Commun. 1979, 90, 925-932.

[23] A. Bridges, L. Gruenke, Y. T. Chang, I. A. Vakser, G. Loew, L. Waskell, J. Biol. Chem. 1998, 273, 17036-17049.

[24] S. Ahuja, N. Jahr, S. C. Im, S. Vivekanandan, N. Popovych, S. V. Le Clair, R. Huang, R. Soong, J. Xu, K. Yamamoto, R. P. Nanga, A. Bridges, L. Waskell, A. Ramamoorthy, J. Biol. Chem. 2013, 288, 22080-22095.

[25] U. H. Durr, K. Yamamoto, S. C. Im, L. Waskell, A. Ramamoorthy, J. Am. Chem. Soc. 2007, 129, 6670-6671.

[26] J. Xu, U. H. Dürr, S. C. Im, Z. Gan, L. Waskell, A. Ramamoorthy, Angew. Chem. Int. Ed. 2008, 47, 7864-7867; Angew. Chem. 2008, 120, 79827985.

[27] M. J. Coon, A. P. Autor, H. W. Strobel, Chem.-Biol. Interact. 1971, 3, 248250. 
[28] M. Ingelman-Sundberg, H. Glaumann, FEBS Lett. 1977, 78, 72-76.

[29] M. Ingelman-Sundberg, H. Glaumann, Biochim. Biophys. Acta Biomembr. 1980, 599, 417-435.

[30] K. Yamamoto, M. Gildenberg, S. Ahuja, S. C. Im, P. Pearcy, L. Waskell, A. Ramamoorthy, Sci. Rep. 2013, 3, 2556.

[31] P. M. Shaw, N. A. Hosea, D. V. Thompson, J. M. Lenius, F. P. Guengerich, Arch. Biochem. Biophys. 1997, 348, 107-115.

[32] J. R. Reed, W. L. Backes, Drug Metab. Rev. 2016, 48, 453-469.

[33] L. Brignac-Huber, J. R. Reed, W. L. Backes, Mol. Pharmacol. 2011, 79, 549-557.

[34] D. R. Davydov, N. Y. Davydova, E. V. Sineva, I. Kufareva, J. R. Halpert, Biochem. J. 2013, 453, 219-230.

[35] K. Marheineke, S. Grünewald, W. Christie, H. Reiländer, FEBS Lett. 1998, $441,49-52$.

[36] Y. Farooq, G. C. Roberts, Biochem. J. 2010, 432, 485-494.

[37] K. C. Liu, J. M. Hughes, S. Hay, N. S. Scrutton, FEBS J. 2017, 284, 2302 2319.

[38] K. Yamamoto, M. A. Caporini, S. C. Im, L. Waskell, A. Ramamoorthy, Biochim. Biophys. Acta Biomembr. 2015, 1848, 342-349.

[39] F. P. Guengerich, M. R. Waterman, M. Egli, Trends Pharmacol. Sci. 2016 37, 625-640.

[40] B. C. Monk, T. M. Tomasiak, M. V. Keniya, F. U. Huschmann, J. D. Tyndall, J. D. O'Connell, R. D. Cannon, J. G. McDonald, A. Rodriguez, J. S. FinerMoore, R. M. Stroud, Proc. Natl. Acad. Sci. USA 2014, 111, 3865-3870.

[41] A. Z. Kijac, Y. Li, S. G. Sligar, C. M. Rienstra, Biochemistry 2007, 46, 13696-13703.

[42] U. H. Dürr, L. Waskell, A. Ramamoorthy, Biochim. Biophys. Acta Biomembr. 2007, 1768, 3235-3259.

[43] U. H. Durr, R. Soong, A. Ramamoorthy, Prog. Nucl. Magn. Reson. Spectrosc. 2013, 69, 1-22.

[44] U. H. Durr, M. Gildenberg, A. Ramamoorthy, Chem. Rev. 2012, 112, 6054-6074.

[45] K. A. Gentry, E. Prade, C. Barnaba, M. Zhang, M. Mahajan, S. C. Im, G. M. Anantharamaiah, S. Nagao, L. Waskell, A. Ramamoorthy, Sci. Rep. 2017, 7, 7793.

[46] M. Zhang, R. Huang, R. Ackermann, S. C. Im, L. Waskell, A. Schwendeman, A. Ramamoorthy, Angew. Chem. Int. Ed. 2016, 55, 4497-4499; Angew. Chem. 2016, 128, 4573-4575.

[47] I. G. Denisov, S. G. Sligar, Chem. Rev. 2017, 117, 4669-4713.

[48] T. H. Bayburt, Y. V. Grinkova, S. G. Sligar, Nano Lett. 2002, 2, 853-856.

[49] I. G. Denisov, Y. V. Grinkova, A. A. Lazarides, S. G. Sligar, J. Am. Chem. Soc. 2004, 126, 3477-3487.

[50] M. Zhang, R. Huang, R. Ackermann, S. C. Im, L. Waskell, A. Schwendeman, A. Ramamoorthy, Angew. Chem. Int. Ed. 2016, 55, 4497-4499; Angew. Chem. 2016, 128, 4573-4575.

[51] H. Kariyazono, R. Nadai, R. Miyajima, Y. Takechi-Haraya, T. Baba, A. Shigenaga, K. Okuhira, A. Otaka, H. Saito, J. Pept. Sci. 2016, 22, 116122.

[52] T. Ravula, C. Barnaba, M. Mahajan, G. M. Anantharamaiah, S. C. Im, L. Waskell, A. Ramamoorthy, Chem. Commun. 2017, 53, 12798-12801.

[53] T. J. Knowles, R. Finka, C. Smith, Y. P. Lin, T. Dafforn, M. Overduin, J. Am Chem. Soc. 2009, 131, 7484-7485.

[54] T. Ravula, N. Z. Hardin, S. K. Ramadugu, S. J. Cox, A. Ramamoorthy, Angew. Chem. Int. Ed. 2018, 57, 1342-1345; Angew. Chem. 2018, 130, 1356-1359.

[55] T. Ravula, N. Z. Hardin, S. K. Ramadugu, A. Ramamoorthy, Langmuir 2017, 33, 10655-10662.

[56] T. Ravula, S. K. Ramadugu, G. Di Mauro, A. Ramamoorthy, Angew. Chem. Int. Ed. 2017, 56, 11466-11470; Angew. Chem. 2017, 129, 11624 11628.

[57] K. Yasuhara, J. Arakida, T. Ravula, S. K. Ramadugu, B. Sahoo, J. I. Kikuchi, A. Ramamoorthy, J. Am. Chem. Soc. 2017, 139, 18657-18663.

[58] M. Šrejber, V. Navrátilová, M. Paloncýová, V. Bazgier, K. Berka, P. Anzenbacher, M. Otyepka, J. Inorg. Biochem. 2018.

[59] M. R. Franklin, R. W. Estabrook, Arch. Biochem. Biophys. 1971, 143, 318329.

[60] J. A. Peterson, R. E. Ebel, D. H. O'Keeffe, T. Matsubara, R. W. Estabrook, J. Biol. Chem. 1976, 251, 4010-4016.

[61] D. R. Davydov, Expert Opin. Drug Metab. Toxicol. 2011, 7, 543-558.

[62] H. Taniguchi, Y. Imai, T. Iyanagi, R. Sato, Biochim. Biophys. Acta Biomembr. 1979, 550, 341-356.

[63] A. Stier, E. Sackmann, Biochim. Biophys. Acta Biomembr. 1973, 311, 400-408.

[64] B. Bösterling, A. Stier, A. Hildebrandt, J. Dawson, J. Trudell, Mol. Pharmacol. 1979, 16, 332-342.
[65] W. G. Balvers, M. G. Boersma, J. Vervoort, A. Ouwehand, I. M. C. M Rietjens, Eur. J. Biochem. 1993, 218, 1021-1029.

[66] R. Narayanasami, J. D. Otvos, C. B. Kasper, A. Shen, J. Rajagopalan, T. J. McCabe, J. R. Okita, D. J. Hanahan, B. S. S. Masters, Biochemistry 1992 31, 4210-4218.

[67] W. D. McClary, J. P. Sumida, M. Scian, L. Paco, W. M. Atkins, Biochemistry 2016, 55, 6258-6268.

[68] J. L. Baylon, I. L. Lenov, S. G. Sligar, E. Tajkhorshid, J. Am. Chem. Soc. 2013, 135, 8542-8551.

[69] D. P. Tieleman, H. J. Berendsen, Biophys. J. 1998, 74, 2786-2801.

[70] G. H. Loew, D. L. Harris, Chem. Rev. 2000, 100, 407-420.

[71] M. Zhang, R. Huang, S. C. Im, L. Waskell, A. Ramamoorthy, J. Biol. Chem. 2015, 290, 12705-12718.

[72] A. N. Volkov, J. A. Worrall, E. Holtzmann, M. Ubbink, Proc. Natl. Acad. Sci. USA 2006, 103, 18945-18950.

[73] S. R. Midtgaard, M. C. Pedersen, J. J. Kirkensgaard, K. K. Sorensen, K. Mortensen, K. J. Jensen, L. Arleth, Soft Matter 2014, 10, 738-752.

[74] R. C. Arenas, B. Danielczak, A. Martel, L. Porcar, C. Breyton, C. Ebel, S. Keller, Sci. Rep. 2017, 7, 45875.

[75] T. Ravula, D. Ishikuro, N. Kodera, T. Ando, G. M. Anantharamaiah, A Ramamoorthy, Chem. Mater. 2018, 30, 3204-3207.

[76] M. Miyazaki, Y. Tajima, T. Handa, M. Nakano, J. Phys. Chem. B. 2010, 114, 12376-12382.

[77] K. L. Browning, T. K. Lind, S. Maric, R. D. Barker, M. Cardenas, M. Malmsten, Colloids Surf. B. Biointerfaces 2018, 168, 117-125.

[78] A. Pralle, P. Keller, E. L. Florin, K. Simons, J. K. H. Hörber, J. Cell Biol. 2000, 148, 997-1008.

[79] E. Sezgin, I. Levental, S. Mayor, C. Eggeling, Nat. Rev. Mol. Cell Biol. 2017, 18, 361-374

[80] J. Fantini, Cell. Mol. Life Sci. 2003, 60, 1027-1032.

[81] R. G. Anderson, K. Jacobson, Science 2002, 296, 1821-1825.

[82] D. Lichtenberg, F. M. Goñi, H. Heerklotz, Trends Biochem. Sci. 2005, 30, 430-436.

[83] R. Shinkyo, F. P. Guengerich, J. Biol. Chem. 2011, 286, 18426-18433.

[84] K. H. Kim, T. Ahn, C. H. Yun, Biochemistry 2003, 42, 15377-15387.

[85] T. Ahn, F. P. Guengerich, C. H. Yun, Biochemistry 1998, 37, 1286012866.

[86] N. A. Treuheit, M. Redhair, H. Kwon, W. D. McClary, M. Guttman, J. P. Sumida, W. M. Atkins, Biochemistry 2016, 55, 1058-1069.

[87] A. Gutteridge, J. Thornton, FEBS Lett. 2004, 567, 67-73.

[88] D. F. Lewis, M. N. Jacobs, M. Dickins, Drug Discovery Today 2004, 9, 530-537.

[89] A. E. Saliba, I. Vonkova, A. C. Gavin, Nat. Rev. Mol. Cell Biol. 2015, 16, 753-761.

[90] M. P. Wymann, R. Schneiter, Nat. Rev. Mol. Cell Biol. 2008, 9, 162-176.

[91] R. M. Epand, Biochim. Biophys. Acta 1998, 1376, 353-368.

[92] T. Harayama, H. Riezman, Nat. Rev. Mol. Cell Biol. 2018, 19, 281-296.

[93] M. Caffrey, Acta Crystallogr Sect B Struct Crystallogr Cryst Chem 2015, 71, 3-18.

[94] E. Nogales, Nature Met. 2015, 13, 24.

[95] K. R. Poudel, D. J. Keller, J. A. Brozik, Soft Matter 2012, 8, 11285.

[96] A. O. Barden, A. S. Goler, S. C. Humphreys, S. Tabatabaei, M. Lochner, M.-D. Ruepp, T. Jack, J. Simonin, A. J. Thompson, J. P. Jones, Neuropharmacology 2015, 98, 22-30.

[97] I. Levental, S. Veatch, J. Mol. Biol. 2016, 428, 4749-4764.

[98] M. Carquin, L. D'Auria, H. Pollet, E. R. Bongarzone, D. Tyteca, Prog. Lipid Res. 2016, 62, 1-24.

[99] S. Fu, S. M. Watkins, G. S. Hotamisligil, Cell Metab. 2012, 15, 623-634.

[100] S. A. Hollingsworth, D. Batabyal, B. D. Nguyen, T. L. Poulos, Proc. Natl. Acad. Sci. USA 2016, 113, 8723-8728.

[101] D. M. Hawcroft, T. W. G. Jones, P. A. Martin, Arch. Gerontol. Geriatr. $1982,1,55-74$.

[102] A. Bindoli, Free Radical Biol. Med. 1988, 5, 247-261.

[103] D. C. Rodriguez Camargo, K. J. Korshavn, A. Jussupow, K. Raltchev, D. Goricanec, M. Fleisch, R. Sarkar, K. Xue, M. Aichler, G. Mettenleiter, A. K Walch, C. Camilloni, F. Hagn, B. Reif, A. Ramamoorthy, Elife 2017, 6, e31226.

[104] D. K. Weber, S. Yao, N. Rojko, G. Anderluh, T. P. Lybrand, M. T. Downton, J. Wagner, F. Separovic, Biophys. J. 2015, 108, 1987-1996.

[105] M. A. Sani, T. C. Whitwell, F. Separovic, Biochim. Biophys. Acta Biomembr. 2012, 1818, 205-211.

[106] M. L. Nasr, D. Baptista, M. Strauss, Z. J. Sun, S. Grigoriu, S. Huser, A. Pluckthun, F. Hagn, T. Walz, J. M. Hogle, G. Wagner, Nat. Methods 2017 $14,49-52$. 
[107] A. Stier, W. Kühnle, R. Rösen, in Microsomes and Drug Oxidations (Eds.: I. Roots, A. Hildebrandt, R. W. Estabrook, A. H. Conney), Pergamon, 1977, pp. 1-8.

[108] U. Akbey, W. T. Franks, A. Linden, S. Lange, R. G. Griffin, B. J. van Rossum, H. Oschkinat, Angew. Chem. Int. Ed. 2010, 49, 7803-7806; Angew. Chem. 2010, 122, 7971-7974.

[109] T. Fujiwara, A. Ramamoorthy, in Annu. Rep. NMR Spectrosc., Vol. 58, Elsevier, 2006, pp. 155-175.

[110] A. Mainz, S. Jehle, B. J. van Rossum, H. Oschkinat, B. Reif, J. Am. Chem. Soc. 2009, 131, 15968-15969.

[111] S. Yan, C. L. Suiter, G. Hou, H. Zhang, T. Polenova, Acc. Chem. Res. 2013, 46, 2047-2058.

[112] R. Zhang, K. H. Mroue, A. Ramamoorthy, Acc. Chem. Res. 2017, 50, 1105-1113.

[113] G. Pintacuda, M. John, X. C. Su, G. Otting, Acc. Chem. Res. 2007, 40, 206-212.

[114] T. Schubeis, T. Le Marchand, L. B. Andreas, G. Pintacuda, J. Magn. Reson. 2018, 287, 140-152.

[115] D. Cala-De Paepe, J. Stanek, K. Jaudzems, K. Tars, L. B. Andreas, G. Pintacuda, Solid State Nucl. Magn. Reson. 2017, 87, 126-136.

[116] J. S. Retel, A. J. Nieuwkoop, M. Hiller, V. A. Higman, E. Barbet-Massin, J. Stanek, L. B. Andreas, W. T. Franks, B. J. van Rossum, K. R. Vinothkumar,
L. Handel, G. G. de Palma, B. Bardiaux, G. Pintacuda, L. Emsley, W. Kuhlbrandt, H. Oschkinat, Nat. Commun. 2017, 8, 2073.

[117] J. Struppe, C. M. Quinn, M. Lu, M. Wang, G. Hou, X. Lu, J. Kraus, L. B. Andreas, J. Stanek, D. Lalli, A. Lesage, G. Pintacuda, W. Maas, A. M. Gronenborn, T. Polenova, Solid State Nucl. Magn. Reson. 2017, 87, 117125.

[118] S. Asami, B. Reif, Acc. Chem. Res. 2013, 46, 2089-2097.

[119] B. Bersch, J. M. Dorr, A. Hessel, J. A. Killian, P. Schanda, Angew. Chem. Int. Ed. 2017, 56, 2508-2512; Angew. Chem. 2017, 129, 2549-2553.

[120] P. Schanda, M. Ernst, Prog. Nucl. Magn. Reson. Spectrosc. 2016, 96, 146.

[121] G. David, M. L. Fogeron, M. Schledorn, R. Montserret, U. Haselmann, S. Penzel, A. Badillo, L. Lecoq, P. Andre, M. Nassal, R. Bartenschlager, B. H. Meier, A. Bockmann, Angew. Chem. Int. Ed. 2018, 57, 4787-4791; Angew. Chem. 2018, 130, 4877-4882.

Manuscript received: May 9, 2018

Accepted Article published: July 11, 2018

Version of record online: July 31, 2018 